\title{
Challenges in exome analysis by LifeScope and its alternative computational pipelines
}

\author{
Erinija Pranckevičiene*, Tautvydas Rančelis, Aidas Pranculis and Vaidutis Kučinskas
}

\begin{abstract}
Background: Every next generation sequencing (NGS) platform relies on proprietary and open source computational tools to analyze sequencing data. NGS tools for Illumina platforms are well documented which is not the case with AB SOLiD systems. We applied several computational and variant calling pipelines to analyse targeted exome sequencing data obtained using AB SOLiD 5500 system. Our investigated tools comprised proprietary LifeScope's pipeline in combination with open source color-space competent mapping programs and a variant caller. We present instrumental details of the pipelines that were used and quantitative comparative analysis of variant lists generated by LifeScope's pipeline versus open source tools.

Results: Sufficient coverage of targeted regions was achieved by all investigated pipelines. High variability was observed in identities of variants across the mapping programs. We observed less than $50 \%$ concordance of variant lists produced by approaches based on different mapping algorithms. We summarized different approaches with regards to coverage (DP) and quality (QUAL) properties of the variants provided by GATK and found that LifeScope's computational pipeline is superior. Fusion of information on mapping profiles (pileup) at genomic positions of variants in several different alignments proved to be a useful strategy to assess questionable singleton variants.
\end{abstract}

Conclusions: We quantitatively supported a conclusion that Lifescope's pipeline is superior for processing sequencing data obtained by AB SOLiD 5500 system. Nevertheless the use of alternative pipelines is encouraged because aggregation of information from other mapping and variant calling approaches helps to resolve questionable calls and increases the confidence of the call. It was noted that a coverage threshold for variant to be considered for further analysis has to be chosen in data-driven way to prevent a loss of important information.

Keywords: LifeScope, Exome analysis pipeline, GATK, Mapping of color-space sequencing data, MAQ, SHRiMP, BFAST, Annovar, Interpretation of genomic variants

\section{Background}

High throughput next-generation sequencing (NGS) has become widely used in practical life-science areas for whole genome and exome sequencing or targeted studies aimed at the identification of deleterious disease-causing genomic variants or in general population genetics studies [1,2]. Accurate interpretation of sequencing results depends on proper laboratory work and a computational pipeline used in the analysis of NGS data. Vendors of each

*Correspondence: erinija.pranckeviciene@mf.vu.lt

Department of Human and Medical Genetics, Faculty of Medicine, Vilnius University, Santariskiu str. 2, LT-08661 Vilnius, Lithuania platform provide proprietary computational software tools to perform analysis of sequencing data obtained from their equipment. In addition, there are many open source programs designed by the research community $[3,4]$. Software tools designed for the Illumina platform are the most documented. Alternatively, software tools for the Life Technologies SOLiD platform are not widely discussed in the scientific literature although SOLiD still appears to be the sequencing platform of choice in many research centers $[5,6]$.

Illumina platform is based on sequencing by synthesis and is using letter-based nucleotide encoding. 
SOLiD platform is employing a different, ligation based, sequencing strategy and uses color-space encoding. In the SOLiD approach each observed DNA base (A, C, $\mathrm{G}$, or $\mathrm{T}$ ) is encoded by a color label defining an order in which two consecutive nucleotides appear in a read [7]. Two-base encoding greatly facilitates identification of sequencing errors because each base is interrogated twice by ligation chemistry. This strategy increases confidence that observed variations at specific genomic locations are true single nucleotide variants.

To align color-space reads to a letter-based reference, the letter-space aligners cannot be used without appropriately transforming either the reads or the reference $[8,9]$. The color-space competent alignment tools often utilized in practice are Blat-like Fast Accurate Search Tool (BFAST), Bowtie, Short Read Mapping Package (SHRiMP) and Mapping and Assembly with Qualities (MAQ) $[10,11]$. However, for mapping and variant calling in SOLiD sequencing data the proprietary LifeScope software by Life Technologies is usually used [5, $12,13]$. Only a very small fraction of reports in literature discussed the LifeScope's pipeline with respect to its alternatives $[5,12]$. Our study attempts to fill this gap by performing a comparative analysis of popular colorspace competent open source tools and the proprietary LifeScope program within a framework of LITGEN project (Genetic diversity of the population of Lithuania and changes of its genetic structure related with evolution and common diseases) [14]. The major contributions of our study consists of:

1. Comparative analysis of the effects of mapping programs on the outcome of variant calling We analyzed color-space competent mapping programs LifeScope, MAQ, SHRiMP and BFAST using near default settings. The mapping programs produce aligned BAM files that are input to a variant calling procedure by GATK. The same variant calling algorithm was applied to all BAM files and produced lists of variants that were different from each other. We aimed to determine the best variant calling approach out of investigated LifeScope, LifeScope-GATK, MAQGATK, BFAST-GATK and SHRiMP-GATK combinations. Schema of our experimental setup is presented in Fig. 1. Quality (QUAL) and coverage depth (DP) of the variant reported by GATK were used as criteria to compare the approaches.

2. Evidence in favor of using an aggregated list of Single Nucleotide Polymorphysms (SNPS) in exomes identified by different approaches in search of possible causative variant-phenotype relationship Annovar was used for variant annotation $[15,16]$. Our analysis of annotated variants led to the conclusion that pooling

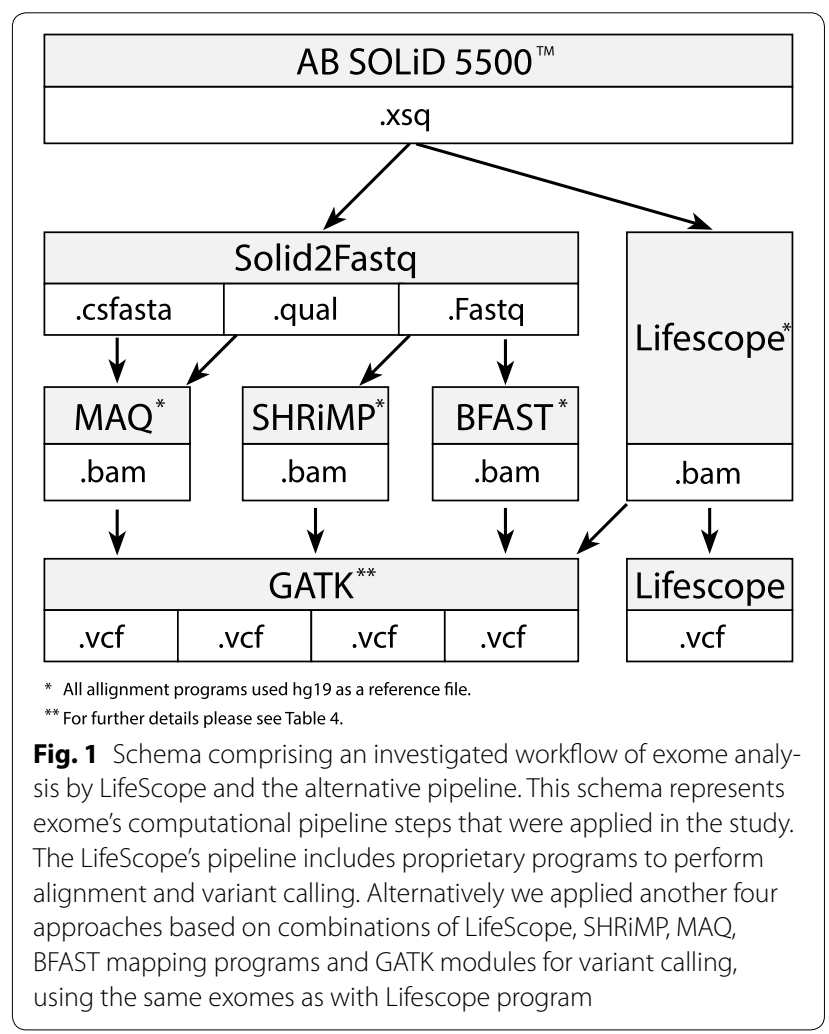

the variants identified by LifeScope and alternative pipelines is more informative in search of possibly damaging variants than using LifeScope's pipeline alone.

3. Detailed description of computational pipeline for analysis of color-space coded targeted exome sequencing data The pipeline comprises all steps from the mapping of raw reads up to the calling of the genomic variants in the exome. We utilize Genome Analysis Toolkit (GATK) for variant calling [17, 18]. GATK tasks and their parameters that worked with LifeScope's BAM files and the alternative to LifeScope mapping programs are described.

Exomes were sequenced at the department of Human and Medical Genetics, Vilnius University with the Life Technologies SOLiD 5500 system using the TargetSeqTM Exome Enrichment Kit without Exact Call Chemistry (ECC). The sequenced fragment read lengths were of $75 \mathrm{bp}$ in color-space coding. A summary statistics of exome sizes of 48 sequenced population samples is as follows: mean $=44.79$ million reads and quartiles were $\mathrm{Q} 25=27.75, \mathrm{Q} 50=40.50$ and $\mathrm{Q} 75=58.50$ million reads. To demonstrate our analysis strategy we use family exomes of proband, father and mother having 63, 31 and 28 million sequenced reads respectively. The targeted 
regions of the exome comprise 195,282 regions in total and consists of $37,268,825$ bases. A workstation and a computing cluster ( 4 nodes, 48 cores) running CentOS 5.6 operating system were used to analyze exomes.

\section{Results Mapping}

It is recommended by GATK creators that $80 \%$ of targeted regions are covered at least by $20 \times$ in order to achieve good results by GATK. In clinical setting it is recommended to have coverage of at least $30 \times$ [19]. The percentages of the target regions in all trio samples covered by $5 \times, 10 \times, 15 \times$ and $20 \times$ by each mapping method are summarized in Table 1 . The best coverage of the target exome regions was achieved by LifeScope and the lowest

Table 1 Percentages of the exome target regions by the mapping programs in family exomes at the cutoffs of $5 x$, $10 x, 15 x$ and $20 x$

\begin{tabular}{lllll}
\hline Coverage & $\mathbf{5 x}(\mathbf{\%})$ & $\mathbf{1 0 \times ( \% )}$ & $\mathbf{1 5 \times ( \% )}$ & $\mathbf{2 0 \times ( \% )}$ \\
\hline LifeScope & 94.28 & 91.29 & 87.69 & 83.56 \\
SHRiMP & 93.97 & 89.92 & 84.94 & 79.07 \\
MAQ & 90.91 & 85.17 & 78.82 & 71.92 \\
BFAST & 86.72 & 78.76 & 70.47 & 62.04 \\
\hline
\end{tabular}

was achieved by BFAST (78 \% by $10 \times$ ) comparing favorably with the coverage in the published exome analysis study of intellectual disability which had $75 \%$ of targeted regions covered at least by $10 \times[20]$.

All mapping methods covered $97 \%$ of the targeted regions. How the methods comapre to each other in mapping is presented in Fig. 2. The largest fraction of the targeted exome regions are covered by 30-60 reads. LifeScope and SHRiMP produced better coverage than MAQ and BFAST. We computed which fraction of regions of the low-coverage (less than 20 reads) by LifeScope are covered better by other mapping programs. SHRiMP improved coverage on $6 \%$ and MAQ on $1 \%$ of those regions. Analysis of the agreement between the individual aligners shows that alternative aligners can map only negligible fraction of the reads unmapped by LifeScope. If compared to each other, then MAQ can map about $28 \%$ of the reads unmapped by BFAST and about $19 \%$ of the reads unmapped by SHRiMP. BFAST can align $12 \%$ and SHRiMP can align $19 \%$ of the reads unmapped by MAQ.

\section{Characterizing different approaches by transition/ transversion $(\mathrm{Ti} / \mathrm{Tv})$ ratio}

To verify if we obtained reliable variant calls we used transition/transversion ratio. This ratio represents a standard to which empirical data should be compared

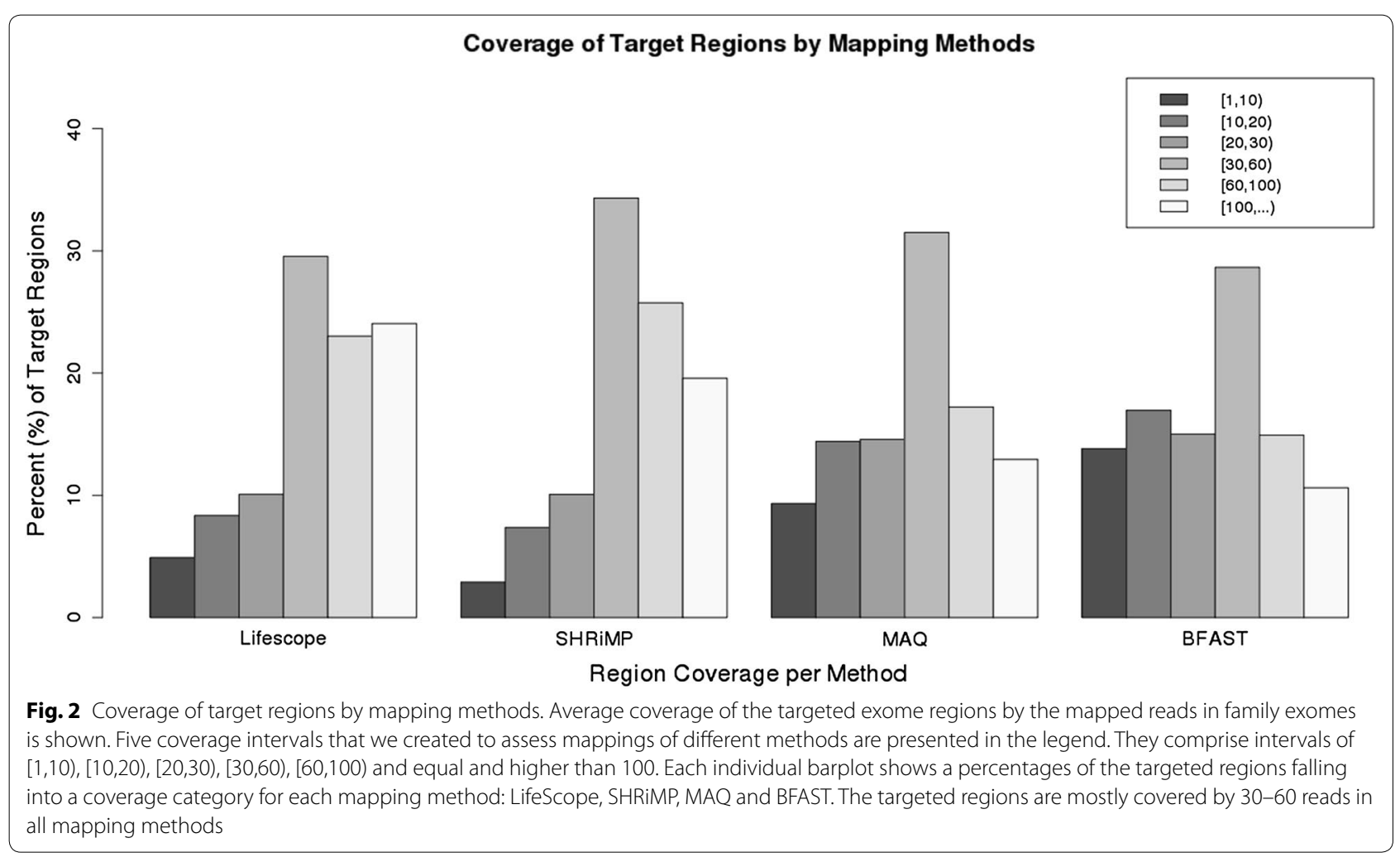


to ensure that the called variants are not random and agree with the natural fundamental variability in human genome. Validated human SNP data showed that a rate between frequency of transitions $(T>C, A>G)$ in genomes to frequency of transversions $(T>A, T>G, C>$ A, C>G) in human genome is 2.1 and in it's coding part (exome) this rate is higher-around 2.8 [17, 21]. If $\mathrm{Ti} / \mathrm{Tv}$ ratio is lower such as $0.5-1$, then it might mean that variants are found at random or there are sequencing artifacts. In our study $\mathrm{Ti} / \mathrm{Tv}$ ratio of variant lists produced by the different approaches indicated that identified variants were not random. $\mathrm{Ti} / \mathrm{Tv}$ ratio in the analyzed exome

Table 2 Transition transversion ratio in different variant calling approaches

\begin{tabular}{llllll}
\hline Approach & LifeScope & $\begin{array}{l}\text { LifeScope- } \\
\text { GATK }\end{array}$ & $\begin{array}{l}\text { MAQ- } \\
\text { GATK }\end{array}$ & $\begin{array}{l}\text { SHRiMP- } \\
\text { GATK }\end{array}$ & $\begin{array}{l}\text { BFAST- } \\
\text { GATK }\end{array}$ \\
\hline $\mathrm{Ti} / \mathrm{TV}$ & 2.62 & 2.45 & 2.59 & 2.3 & 2.68 \\
\hline
\end{tabular}

data by different methods was close to $2.2-2.7$ as shown in Table 2. The agreement between the methods with respect to $\mathrm{Ti} / \mathrm{Tv}$ ratio was high.

\section{Variant calling results}

Regardless of a targeted nature of exome sequencing experiment important variants can be found outside the boundaries of the targeted regions [22]. Therefore we used all identified variants in our study. It is expected that majority of the identified variants have already been found and documented in public databases. We assessed how many variants found in the analyzed exomes are present in dbSNP, 1000Genomes and ESP6500 databases. These counts are shown in Table 3. Most of the identified variants (96-98\%) were included in dbSNP138, thus validating the used approaches. Largest proportions of variants were found by LifeScope-GATK and SHRiMPGATK. The least number of variants was found by BFAST-GATK. A proportion of variants found by each

Table 3 Counts of SNPs identified in the family exomes present in the datasets of known variants

\begin{tabular}{|c|c|c|c|c|c|}
\hline & LifeScope & LifeScope-GATK & MAQ-GATK & SHRiMP-GATK & BFAST-GATK \\
\hline \multicolumn{6}{|l|}{ Proband } \\
\hline Total & 38,626 & 60,313 & 49,483 & 67,669 & 43,536 \\
\hline dbsnp138 & 37,312 & 58,342 & 48,538 & 65,185 & 42,909 \\
\hline $1000 G$ & 35,511 & 54,745 & 46,706 & 62,778 & 42,030 \\
\hline esp6500 & 31,319 & 29,745 & 26,041 & 27,386 & 25,169 \\
\hline In target regions & 21,837 & 19,530 & 16,958 & 17,082 & 15,856 \\
\hline dbsnp138 & 20,892 & 18,869 & 16,707 & 16,768 & 15,688 \\
\hline $1000 G$ & 19,504 & 17,954 & 16,260 & 16,304 & 15,321 \\
\hline esp6500 & 17,894 & 16,469 & 14,912 & 14,891 & 14,138 \\
\hline \multicolumn{6}{|l|}{ Mother } \\
\hline Total & 35,267 & 43,831 & 28,032 & 38,711 & 26,898 \\
\hline dbsnp138 & 34,075 & 42,778 & 27,641 & 38,337 & 26,626 \\
\hline $1000 G$ & 32,455 & 40,316 & 26,472 & 36,856 & 26,170 \\
\hline esp6500 & 29,095 & 26,101 & 17,909 & 23,348 & 19,139 \\
\hline In target regions & 20,995 & 17,697 & 12,566 & 15,477 & 12,722 \\
\hline dbsnp138 & 20,099 & 17,296 & 12,470 & 15,359 & 12,623 \\
\hline $1000 G$ & 18,806 & 16,459 & 12,143 & 14,939 & 12,384 \\
\hline esp6500 & 17,281 & 15,099 & 11,163 & 13,658 & 11,469 \\
\hline \multicolumn{6}{|l|}{ Father } \\
\hline Total & 36,350 & 46,822 & 36,798 & 42,486 & 27,949 \\
\hline dbsnp138 & 35,106 & 45,723 & 36,209 & 42,193 & 27,669 \\
\hline $1000 G$ & 33,381 & 42,939 & 34,777 & 40,782 & 27,204 \\
\hline esp6500 & 29,765 & 26,728 & 22,177 & 24,458 & 19,715 \\
\hline In target regions & 21,254 & 17,936 & 14,972 & 15,760 & 13,033 \\
\hline dbsnp138 & 20,348 & 17,531 & 14,827 & 15,661 & 12,931 \\
\hline $1000 G$ & 19,015 & 16,668 & 14,447 & 15,296 & 12,692 \\
\hline esp6500 & 17,457 & 15,323 & 13,326 & 14,042 & 11,756 \\
\hline
\end{tabular}

For each exome and the approach a total number of all identified SNPs and the SNPs that are only in the targeted regions are shown in italic 
method correlates with the number of original reads available for mapping and also the mapping efficiency of the alignment program.

We observed a moderate concordance between the variant lists. The concordance was measured by counting overlapping genomic positions of variants between the methods computed by intersect tool in BEDtools [23]. Table 4 shows numbers and fractions of variants found by all approaches and singleton variants specific to methods. All methods agree on $30-50 \%$ of identified variants. This is consistent with the results of the study in which a concordance in variant calling approaches was investigated for Illumina platform and it was found to be less than $60 \%$ [24]. LifeScope and LifeScope-GATK consistently identified around $10 \%$ of variants unique only to those methods. In MAQ-GATK approach this fraction was around $4 \%$. Fractions of method-specific variants in SHRiMP-GATK and BFAST-GATK are not consistent across the exomes. Variability in variant identities across the methods arises due to differences in BAM files caused by differences in mapping algorithms and mapping qualities.

Depth of coverage (DP) and variant quality (QUAL) properties are assigned by GATK to the called variants. DP represents number of reads that overlap in the genomic position of a variant. QUAL is Phred encoded score assigned to the variant by GATK showing call quality and it can be very large. We assume that a better variant calling approach produces variants with high DP and QUAL values. To compare the DP and QUAL across the methods we used the variants simultaneously identified by all used methods. Figure 3 illustrates per-method per exome differences in QUAL property by means of its empirical distribution functions. The best QUAL values were achieved by SHRiMP-GATK, followed by
MAQ-GATK, followed by LifeScope-GATK and the last was BFAST-GATK. The overall result for the DP property is presented in Table 5. With regards to DP a highest variant coverage is achieved by LifeScope. Variants produced by both SHRiMP-GATK and LifeScope-GATK have higher median coverage than MAQ-GATK and BFAST-GATK.

With respect to both DP and QUAL properties of the variant, SHRiMP-GATK ranks first and LifeScope-GATK second. Variant calls by MAQ-GATK do not have high coverage (DP value) nevertheless they have high quality. In proband exome $46.54 \%$ of the calls had higher or equal DP in BFAST-GATK versus $53.46 \%$ of the calls in LifeScope-GATK, in the mother exome it was 25.02 versus $74.98 \%$ and in the father exome it was 27.32 versus $72.68 \%$. Overall results that we observed suggest that LifeScope mapping program is superior in mapping color-space data.

\section{Variant annotation}

Variant annotations have been performed using Annovar [15]. It is the most widely used software tool for interpretation of genomic variants found in high-throughput sequencing data. Annotation was performed using table annovar.pl script, which generates an Excel compatible file with integrated information for a given list of variants. For variant interpretation it is important that the used pipeline identifies as many deleterious variants as possible.

ClinVar is a public archive that provides reports of relationships among medically important variants and phenotypes. Data to ClinVar streams from OMIM, GeneReviews, dbSNP and also from direct submissions by scientists. Database represents 19,774 genes which include 149,202 variants from 248 submitters [25].

Table 4 Agreement between variant calling approaches on all called SNPs

\begin{tabular}{|c|c|c|c|}
\hline Exome & Proband & Mother & Father \\
\hline Total number of SNP variants union of all approaches & 86,840 & 55,738 & 68,915 \\
\hline Number of SNP variants identified by all approaches common & 21,687 & 13,995 & 11,483 \\
\hline Fraction of common variants in total identified by LifeScope (\%) & 56 & 40 & 32 \\
\hline LifeScope-GATK (\%) & 32 & 44 & 25 \\
\hline MAQ-GATK (\%) & 44 & 50 & 31 \\
\hline SHRIMP-GATK (\%) & 32 & 36 & 27 \\
\hline BFAST-GATK (\%) & 50 & 52 & 41 \\
\hline Number of SNP variants and fraction of total specific to LifeScope & $2936(7.6 \%)$ & $4543(12.9 \%)$ & $4246(11.7 \%)$ \\
\hline LifeScope-GATK & $5251(8.7 \%)$ & $4468(10.2 \%)$ & $4555(9.7 \%)$ \\
\hline MAQ-GATK & $1943(3.9 \%)$ & $1074(3.8 \%)$ & $1369(3.7 \%)$ \\
\hline SHRIMP-GATK & $14,276(21.1 \%)$ & $2210(5.7 \%)$ & 1644 (3.9\%) \\
\hline BFAST-GATK & 1097 (2.5\%) & $574(2.1 \%)$ & 9851 (35.2\%) \\
\hline
\end{tabular}

By common are denoted variants that have been identified by all approaches. By specific are identified variants that were identified exclusively by one approach. The percentages are computed as fraction of the total number of all variants shown in Table 3 identified by that method 

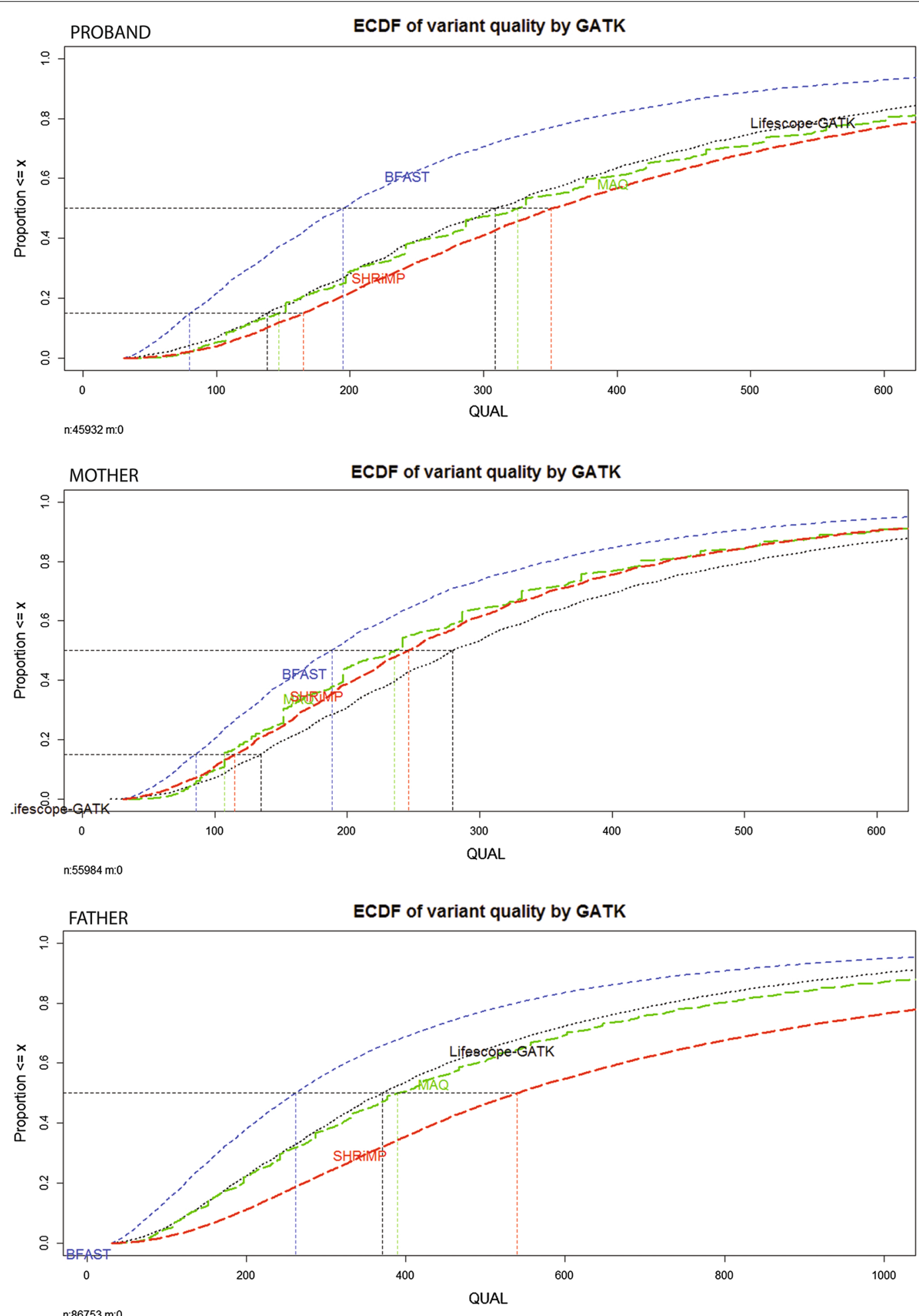

Fig. 3 Empirical cumulative distribution function (ECDF) of variant quality (QUAL) property assigned by GATK for variants identified in alignments produced by different mapping programs. To compute ECFD only variants that have been identified by all approaches together were used. ECDF's of different alignments are color-coded: BFAST by blue line, LifeScope-GATK by black, MAQ by green and SHRiMP by red. Panels correspond to the family exomes. ECDF plots of QUAL per method in proband exome are on the top, the mother exome is in the middle and exome of the father is on the bottom. Median QUAL value of LifeScope-GATK consistently apears around 300 in all exomes. For BFAST-GATK it is around 200. Other approaches differ across the exomes 
Table 5 Summary of coverage [depth of coverage (DP) assigned to a variant by GATK] for variants called by the different approaches in family exomes

\begin{tabular}{|c|c|c|c|c|c|c|c|}
\hline \multirow{2}{*}{$\begin{array}{l}\text { Proband variants } \\
\text { Identification method }\end{array}$} & \multicolumn{3}{|c|}{ All variants per method } & \multicolumn{2}{|c|}{ Common 21,687 variants } & \multicolumn{2}{|l|}{ Variants in COSMIC } \\
\hline & $\begin{array}{l}\text { Coverage quartiles } \\
\text { Q25,Med,Q75 }\end{array}$ & Total \# & $\begin{array}{l}\text { Sites } \% \\
\geq 8 x\end{array}$ & $\begin{array}{l}\text { Coverage quartiles } \\
\text { Q25,Med,Q75 }\end{array}$ & $\begin{array}{l}\text { Sites } \% \\
\geq 8 x\end{array}$ & $\begin{array}{l}\text { Coverage quartiles } \\
\text { Q25,Med,Q75 }\end{array}$ & Total \# \\
\hline LifeScope & $12,25,46$ & 38,626 & 87 & $16,28,46$ & 92 & $11,28,51$ & 89 \\
\hline LifeScope-GATK & $7,14,24$ & 60,313 & 75 & $14,21,30$ & 95 & $16,22,35$ & 21 \\
\hline MAQ-GATK & $7,13,23$ & 49,483 & 74 & $10,17,25$ & 86 & $25,33,64$ & 15 \\
\hline SHRIMP-GATK & $6,16,34$ & 67,669 & 72 & $21,32,50$ & 98 & $15,52,120$ & 22 \\
\hline BFAST-GATK & $7,13,22$ & 43,536 & 73 & $11,18,27$ & 89 & $7,8,10$ & 10 \\
\hline \multirow{2}{*}{$\begin{array}{l}\text { Mother variants } \\
\text { Identification method }\end{array}$} & \multicolumn{3}{|c|}{ All variants per method } & \multicolumn{2}{|c|}{ Common 13,995 variants } & \multicolumn{2}{|l|}{ Variants in COSMIC } \\
\hline & $\begin{array}{l}\text { Coverage quartiles } \\
\text { Q25,Med,Q75 }\end{array}$ & Total \# & $\begin{array}{l}\text { Sites } \% \\
\geq 8 x\end{array}$ & $\begin{array}{l}\text { Coverage quartiles } \\
\text { Q25,Med,Q75 }\end{array}$ & $\begin{array}{l}\text { Sites } \% \\
\geq 8 \times\end{array}$ & $\begin{array}{l}\text { Coverage quartiles } \\
\text { Q25,Med,Q75 }\end{array}$ & Total \# \\
\hline LifeScope & $8,15,26$ & 35,267 & 76 & $12,20,30$ & 89 & $7,16,28$ & 101 \\
\hline LifeScope-GATK & $6,10,16$ & 43,831 & 65 & $10,15,21$ & 90 & $10,23,27$ & 13 \\
\hline MAQ-GATK & $6,10,15$ & 28,032 & 61 & $6,10,15$ & 66 & $25,32,61$ & 6 \\
\hline SHRIMP-GATK & $6,11,17$ & 38,711 & 68 & $10,14,20$ & 88 & $17,20,34$ & 13 \\
\hline BFAST-GATK & $5,9,14$ & 26,898 & 58 & $2,12,16$ & 74 & $5,6,8.5$ & 6 \\
\hline \multirow{2}{*}{$\begin{array}{l}\text { Father variants } \\
\text { Identification method }\end{array}$} & \multicolumn{3}{|c|}{ All variants per method } & \multicolumn{2}{|c|}{ Common 11,483 variants } & \multicolumn{2}{|l|}{ Variants in COSMIC } \\
\hline & $\begin{array}{l}\text { Coverage quartiles } \\
\text { Q25,Med,Q75 }\end{array}$ & Total \# & $\begin{array}{l}\text { Sites } \% \\
\geq 8 x\end{array}$ & $\begin{array}{l}\text { Coverage quartiles } \\
\text { Q25,Med,Q75 }\end{array}$ & $\begin{array}{l}\text { Sites } \% \\
\geq 8 x\end{array}$ & $\begin{array}{l}\text { Coverage quartiles } \\
\text { Q25,Med,Q75 }\end{array}$ & Total \# \\
\hline LifeScope & $8,16,29$ & 36,350 & 78 & $12,21,31$ & 89 & $8,15,31.5$ & 99 \\
\hline LifeScope-GATK & $6,11,18$ & 46,822 & 68 & $11,16,22$ & 90 & $14,23,30$ & 12 \\
\hline MAQ-GATK & $6,10,17$ & 36,798 & 66 & $8,12,18$ & 76 & $34,57,94$ & 5 \\
\hline SHRiMP-GATK & $7,12,20$ & 42,486 & 74 & $12,18,25$ & 94 & $17,21,21$ & 4 \\
\hline BFAST-GATK & $6,9,15$ & 27,949 & 61 & $7,11,17$ & 73 & $8,12,17$ & 93 \\
\hline
\end{tabular}

Coverage is summarized as median and 1 st and $3 r d$ quartile showing a central tendency. This coverage tendency is presented for: all called variants, common variants called by all methods and approach-specific variants in COSMIC. An additional quality measure-a fraction of variants-covered at least by 8 reads is shown for each method for all and common variants

COSMIC (Catalog of Somatic Mutations in Cancer) database is designed to store and display somatic mutation information and contains information relating to human cancers - publications, samples and mutations. COSMIC database describes over 2500 cancer disease classifications, from 47 primary tissue types and represents full literature curation of 136 genes and 12,542 cancer genomes [26]. We analyzed how many harmful variants from ClinVar and COSMIC were identified by each investigated variant calling approach and how these approaches complemented each other in detecting important deleterious variants. Summary of this analysis is presented in Table 6. Figure 4 illustrates an agreement of the variant calling approaches in detecting those deleterious variants. Largest number of deleterious variants is detected by LifeScope's pipeline. Pipelines based on MAQ and BFAST are similar to each other in terms of their performance.

A variant of high confidence has to have high coverage. Table 6 presents calculation of information loss if only taking into consideration the deleterious variants covered by $15 \times$. About $\sim 10-15 \times$ is assumed to be a minimum coverage for a confident call [20]. Our results show that in cases of exomes having smaller coverage high-threshold coverage filters may lead to information losses up to $50 \%$. Lesser loss was noted in LifeScope's pipeline. The biggest loss of detected variants by constraining the coverage was in BFAST-GATK and MAQ-GATK approaches.

Overlap of the methods in terms of their detected deleterious variants is illustrated in Fig. 4. Almost every pathogenic and drug response variant was identified by either one or several out of four alternative approaches and LifeScope. Few singleton pathogenic variants were specific to LifeScope and LifeScope-GATK pipelines. Just a single variant was detected solely by MAQ-GATK. Comparison of approaches with respect to variants in COSMIC showed that there are cases in which alternative pipelines are calling an important variant, which did not gain enough coverage or quality during the processing by other pipelines. Use of several alternative variant calling pipelines helps to resolve questionable variant calls. 


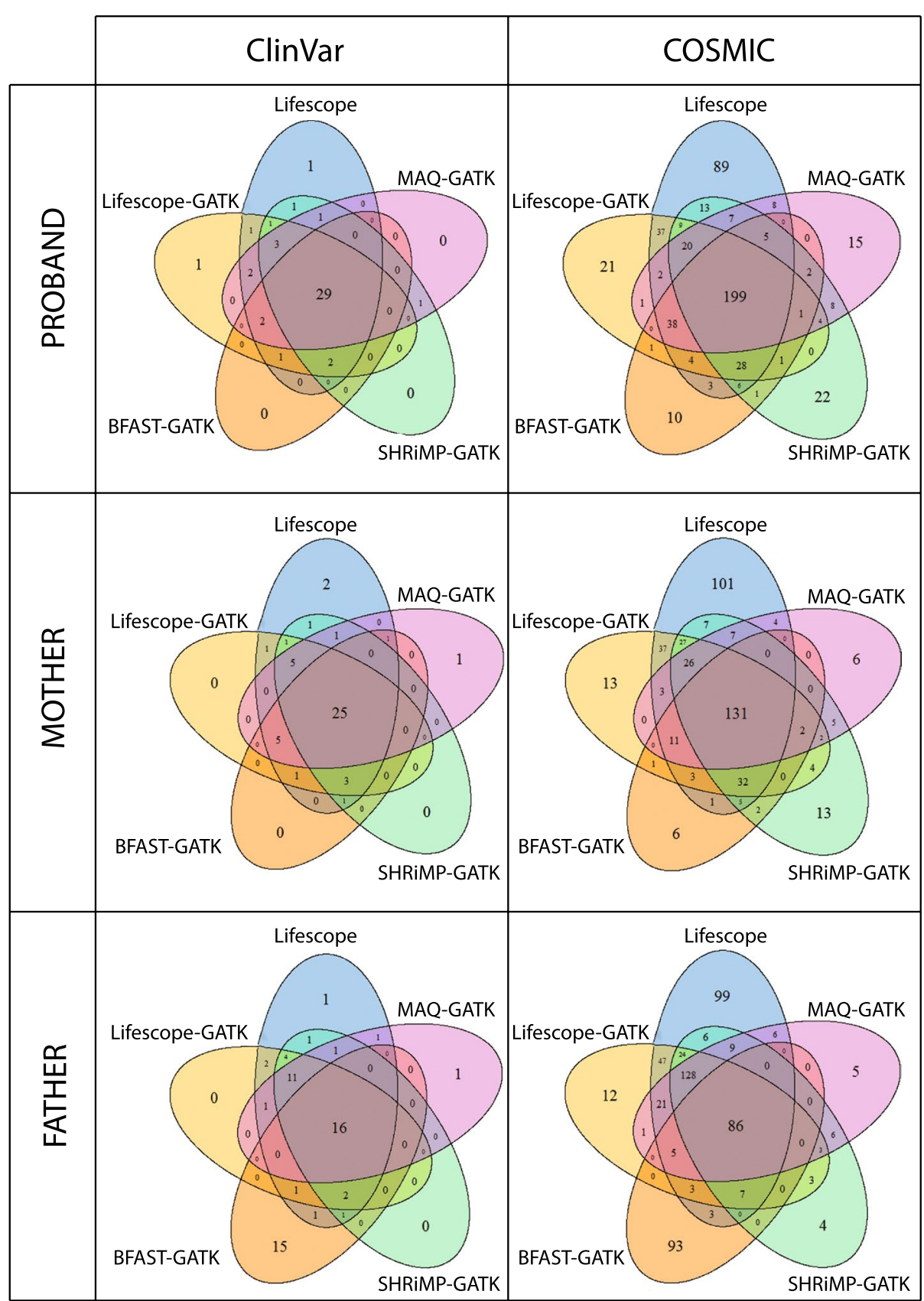

Fig. 4 Agreement between different variant calling approaches with respect to called SNPs that are present in ClinVar and COSMIC databases. Venn diagrams show how much different approaches agree in identifying harmful variants. The middle area of each diagram shows number of variants common to all methods. The Venn diagram leafs show number of variants specific to each method. On the left are diagrams representing SNPs in ClinVar database and on the right is distribution of identified SNPs present in COSMIC database. The ittop part of the figure shows diagrams of proband, the itmiddle represents mother and the bottom represents father. Substantial agreement between the methods was observed on pathogenic and drug response ClinVar variants

\section{Differences between the used variant calling approaches} We compared performance of proprietary versus open source based pipeline in exome analysis using a near default setting and observed only moderate agreement of different methods on SNP calls. Similar agreement of variant calling pipelines on data produced by Illumina platform was already noted [24]. It can be argued that the agreement of different methods with respect to SNP calls 
Table 6 SNPs annotated in ClinVar and COSMIC databases per method and per person

\begin{tabular}{|c|c|c|c|c|}
\hline & LifeScope & LifeScope-GATK & MAQ-GATK & BFAST-GATK \\
\hline \multicolumn{5}{|l|}{ Proband } \\
\hline ClinVar total & 1328 & 1261 & 1116 & 1150 \\
\hline Deleterious variants & 44;34pat + 10drug & 42;33pat + 9drug & 38;29pat + 9drug & $34 ; 27$ pat +7 drug \\
\hline Deleterious at $15 \times$ & $32 ; 23 p a t+9 d r u g$ & 38;28pat + 10drug & 24;19pat + 5drug & $21 ; 15$ pat + 6drug \\
\hline Loss going to $15 x$ & $27 \%$ & $9.5 \%$ & $37 \%$ & $38 \%$ \\
\hline COSMIC total & 468 & 366 & 310 & 299 \\
\hline COSMIC variants at $15 \times$ & 349 & 238 & 195 & 168 \\
\hline Loss going to $15 \times$ & $25 \%$ & $35 \%$ & $37 \%$ & $44 \%$ \\
\hline \multicolumn{5}{|l|}{ Mother } \\
\hline ClinVar total & 1288 & 1170 & 841 & 947 \\
\hline Deleterious variants & 47;38pat + 9drug & 41;33pat + 8drug & 38;30pat + 8drug & 36;30pat + 6drug \\
\hline Deleterious at $15 \times$ & $20 ; 15$ pat + 5drug & $30 ; 24$ pat + 6drug & 11;10pat + 1drug & $11 ; 8$ pat +3 drug \\
\hline Loss going to $15 \times$ & $57 \%$ & $27 \%$ & $71 \%$ & $69 \%$ \\
\hline COSMIC total & 395 & 292 & 197 & 194 \\
\hline $\cos M I C$ at $15 x$ & 231 & 124 & 73 & 63 \\
\hline Loss going to $15 \times$ & $42 \%$ & $52 \%$ & $63 \%$ & $68 \%$ \\
\hline \multicolumn{5}{|l|}{ Father } \\
\hline ClinVar total & 1248 & 1126 & 950 & 959 \\
\hline Deleterious variants & 43;36pat + 7drug & $37 ; 31$ pat + 6drug & $31 ; 26$ pat + 5drug & 36;30pat + 6drug \\
\hline Deleterious at $15 \times$ & 19;16pat + 3drug & 26;22pat + 4drug & 12;11pat + 1drug & 11;9pat + 2drug \\
\hline Loss going to $15 \times$ & $56 \%$ & $30 \%$ & $61 \%$ & $69 \%$ \\
\hline COSMIC total & 444 & 340 & 270 & 197 \\
\hline $\cos M I C$ at $15 x$ & 288 & 176 & 122 & 74 \\
\hline Loss going to $15 x$ & $35 \%$ & $37 \%$ & $55 \%$ & $62 \%$ \\
\hline
\end{tabular}

Total number of identified variants in ClinVar is shown together with the number of patogenic (pat) and drug response (drug) variants. Counts of high confidence variants covered at least by $15 \times$ are presented for both: ClinVar and COSMIC. A fraction of total variants which would be lost by going to a higher coverage is presented by a percentage

can be improved by parameter tuning in mapping and variant calling phases.

Ideally for the purposes of comparison, the compatible parameters of mapping programs would be set to equal values to generate BAM files for variant calling. Such equalization is very difficult in practice and may require a reverse-engineering of the mapping programs. LifeScope operates using dozens of parameters. A majority of these parameters do not have obvious counterparts in the open source aligners. Other variation which is difficult to control arises from indexing. In BFAST and SHRiMP indexing of the reference by default spaced seeds will be different from the indexing scheme used by LifeScope leading to differing alignment of reads in BAM files affecting the variant calling. Variant calling features affected by mapping can be explored through the GATK variant annotations.

Using variants identified by HaplotypeCaller (see step 8 in Table 7) GATK builds a confident variant call prototype based on a multivariate Gaussian mixture modelling. Model parameters are estimated using variant annotations computed from data in BAM file of called variants present in the dbSNP database of known variants. Final variants are called by applying GATK VariantRecalibrator task (see step 9 in Table 7). Variant annotationsquality (qual), depth (DP), Fisher Strand (FS), root mean square of Mapping Quality of reads supporting a variant call (MQ), quality by depth (QD), Mann-Whitney-Wilcoxon Rank Sum tests MQRankSum, ReadPosRankSum, BaseQRankSum and ClippingRankSum-characterize low level properties of variants from information in BAM file. By comparing values of these annotations the major differences between variants identified by different approaches ( Lifescope-GATK, MAQ-GATK, BFASTGATK and SHRiMP-GATK) can be delineated. In our study, a large fraction of variants identified only by a single approach had low quality (GATK LowQual filter value).

Which of variant annotations discriminate the variant classes the best, was explored using common variants by C5.0 decision tree algorithm [28] in R. We do not attempt to fit a classification model but rather to perform exploratory analysis to discover thresholds of variant annotations best discriminating variant classes. C5.0 algorithm 
learns this information from data. A most discriminative annotation was root mean square mapping quality value (MQ). Almost all SHRiMP-GATK variants were assigned to a class characterized by high MQ (>99). Mapping quality computation is specific to each mapping program. Therefore, MQ values might not be directly comparable between the approaches. MQ and MQRankSum were exluded from the exploratory list of annotations and the additional sets of rules discriminating the variants were identified. Class of SHRiMP-GATK variants had a larger value of depth of coverage (DP). Class of MAQ-GATK variants was characterized by higher value of quality by depth (QD) and better quality assigned by GATK. One subclass of Lifescope-GATK variants had higher QD values. Another subclass of Lifescope-GATK variants had lower QD and a negative BaseQRankSum, indicating poorer base quality support for alternative alleles. The diagram of a decision tree of these classifications is shown in Fig. 5.

Using LifeScope BAM files, a variant calling was performed by both LifeScope and GATK. Some variants were identified solely by LifeScope and vice versa. In the GATK variant calling pipeline an adjustment of reported base qualities in BAM file is performed according to the estimated empirical base quality scores (see step 8 in Table 7). We observed that some base qualities reported in LifeScope's BAM file were diminished and some were elevated by GATK's base recalibration procedure. This adjustment resulted in some sites called variants by GATK but not by LifeScope and vice versa. The sites called variants by LifeScope were not recognized by GATK because of reduction of base quality scores resulting from the base recalibration. Default variant calling parameters of LifeScope are less stringent (for example a minimum coverage for Heterozygote call is 2). Therefore such variants identified by LifeScope are filtered by GATK.

\section{Shortcomings of used alignment programs}

Our aim was to assess performances of the tools with the default parameters. A major shortcoming of MAQ was rather long time required to complete analysis since MAQ was not designed to utilize multicore computing resources. MAQ and BFAST generally produced lower coverage of targeted regions. However, their alignments had higher mapping quality overall. SHRiMP was the best in terms of speed and coverage. However, with the default parameters SHRiMP produced alignments characterized

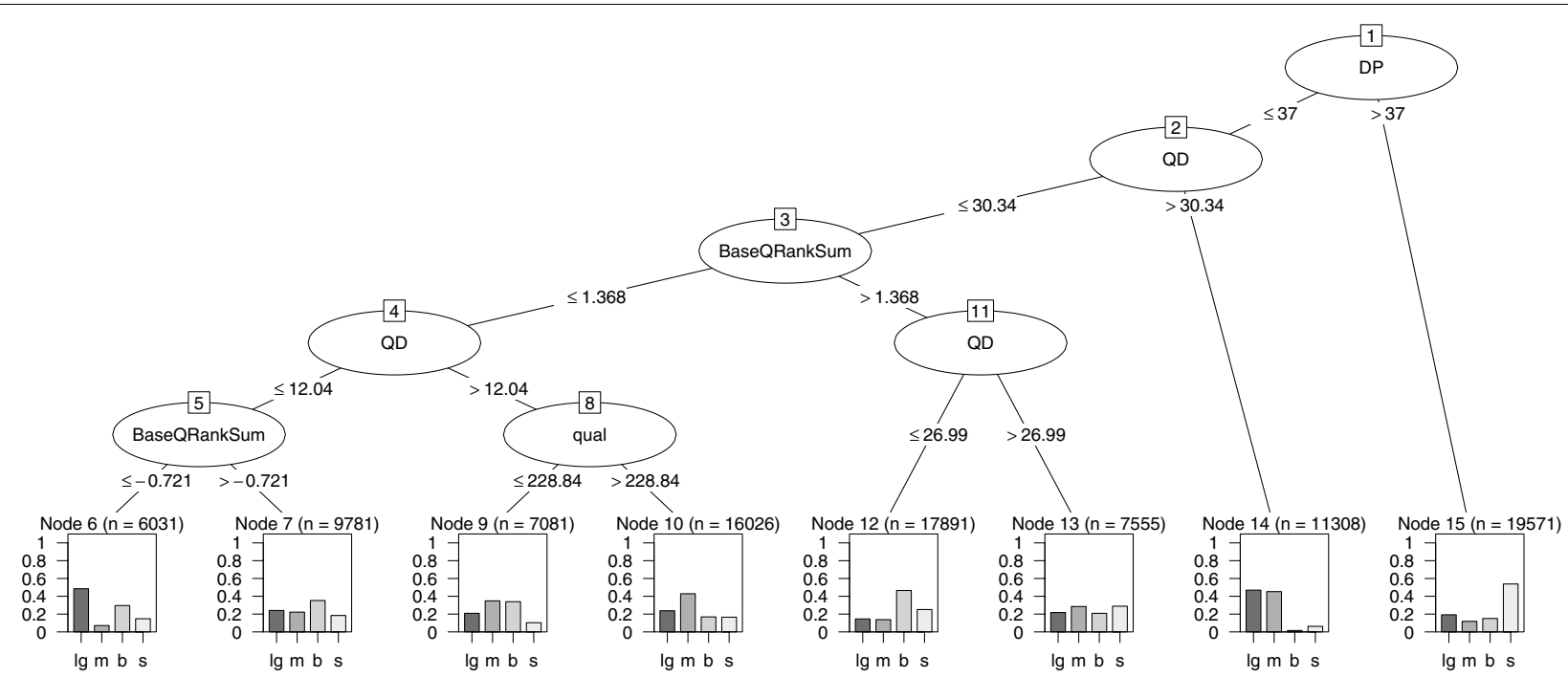

Fig. 5 Decision tree diagram showing most discriminative annotations in classification of different categories of common variants identified in proband. Rectangular terminal nodes indicate fractions of variants (percentage) classified by the rules in each branch of the binary tree. Variable $n$ indicates the number of samples from the training set assigned to that node. Node is associated with a class label of the most prevalent variant class. For example node 15 is associated with SHRiMP-GATK variants. Variant class identified by LifeScope-GATK is denoted by lg; SHRiMP-GATK is denoted by s; MAQ-GATK is denoted by $m$ and BFAST-GATK by $b$. Tree nodes represented by ellipses show GATK variant annotations which were the most important in classifying the variants at each subsequent level. Classification rules are indicated by less or equal than and greater than conditions applied on a threshold value of the parameter. The diagram shows a considerable fraction of SHRiMP-GATK variants in node 15 characterized by larger depth of coverage (DP > 37). Large fraction of MAQ-GATK $(m)$ variants are characterized by higher values of quality by depth (QD) (node 14) and have better GATK-assigned quality (see node 10). A group of LifeScope-GATK (Ig) variants (node 14) are characterized by higher quality by depth (QD > 30.34). Another group (node 6) has lower QD and a negative value of BaseQRankSum, indicating poorer base quality support for alternative alleles. A tree size while running C50 algorithm was controlled constraining a split by minimum number of cases (parameter minCases) equal to 5000 
Table 7 GATK steps

\begin{tabular}{|c|c|c|}
\hline Step & Command & Description \\
\hline Input BAM & $\begin{array}{l}\text { java -jar MarkDuplicates.jar INPUT = your_bam_file OUTPUT }=\text { step1.bam METRICS_FILE }=\text { Fmetrics_ } \\
\text { step 1.bam ASSUME_SORTED = true }\end{array}$ & Marking duplicates \\
\hline Step 1. & $\begin{array}{l}\text { java -jar AddOrReplaceReadGroups.jar INPUT= step1.bam OUTPUT = step2.bam RGID= Read_Group } \\
I D R G L B=\text { Read_Group_Library RGPL= platform RGPU = platform_unit RGSM = sample_name } \\
R G D S=\text { Read_Group_Description RGDT = Read_Group_Run_Date }\end{array}$ & $\begin{array}{l}\text { Replacing all read groups in the } \\
\text { INPUT file with a new read group }\end{array}$ \\
\hline Step 2. & java -jar ReorderSam.jar INPUT $=$ step2.bam OUTPUT $=$ step3.bam REFERENCE $=$ ucsc.hg 19.fasta & $\begin{array}{l}\text { Reorder reads in BAM file to match } \\
\text { the contig ordering in a provided } \\
\text { reference file }\end{array}$ \\
\hline Step 3. & java -jar SortSam.jar INPUT = step3.bam OUTPUT = step4.bam SORT_ORDER $=$ coordinate & $\begin{array}{l}\text { Sorting the aligned reads by coordi- } \\
\text { nate order }\end{array}$ \\
\hline \multirow[t]{3}{*}{ Step 4.} & java-jar BuildBamIndex.jar INPUT= step4.bam & Generating BAM index \\
\hline & $\begin{array}{l}\text { java-jar GenomeAnalysisTK.jar-TRealignerTargetCreator-R ucsc.hg 19.fasta-S STRICT -I step4.bam -o } \\
\text { indels.intervals -allowPotentiallyMisencodedQuals }\end{array}$ & $\begin{array}{l}\text { Indel Realignment I (Creating a tar- } \\
\text { get list of intervals to be realigned) }\end{array}$ \\
\hline & $\begin{array}{l}\text { java-jar GenomeAnalysisTK.jar -TIndelRealigner-R ucsc.hg19.fasta-S STRICT-I step4.bam-targetIntervals } \\
\text { indels.intervals -o step5.bam-known Mills_and_1000G_gold_standard.indels.hy19.vcf-known 1000G_ } \\
\text { phase1.indels.hy 19.vcf-allowPotentiallyMisencodedQuals }\end{array}$ & $\begin{array}{l}\text { Indel Realignment II (Performing } \\
\text { realignment of the target intervals) }\end{array}$ \\
\hline Step 5. & java -jar SortSam.jar INPUT = step5.bam OUTPUT = step6.bam SORT_ORDER $=$ coordinate & $\begin{array}{l}\text { Sorting the aligned reads by coordi- } \\
\text { nate order }\end{array}$ \\
\hline \multirow[t]{3}{*}{ Step 6.} & java-jar BuildBamIndex.jar INPUT = step6.bam & Generating BAM index \\
\hline & $\begin{array}{l}\text { java-jar GenomeAnalysisTK.jar -T BaseRecalibrator-I step6.bam -R ucsc.hg 19.fasta-S STRICT-knownSites } \\
\text { dbsnp_138.hg19.vcf-o recal.grp-covariate QualityScoreCovariate -covariate ReadGroupCovariate } \\
\text {-covariateContextCovariate-covariate CycleCovariate -solid_nocall_strategy PURGE_READ -solid_ } \\
\text { recal_modeSET_Q_ZERO_BASE_N-allowPotentiallyMisencodedQuals }\end{array}$ & $\begin{array}{l}\text { Base quality score recalibration I } \\
\text { (data-driven adjustment of base } \\
\text { quality scores) }\end{array}$ \\
\hline & $\begin{array}{l}\text { java -jar GenomeAnalysisTK.jar -R ucsc.hg 19.fasta-S STRICT -I step6.bam -T PrintReads -o step7.bam } \\
\text {-BQSR recal.grp-allowPotentiallyMisencodedQuals }\end{array}$ & $\begin{array}{l}\text { Base quality score recalibration II } \\
\text { (Applying the recalibration to } \\
\text { sequence data) }\end{array}$ \\
\hline Step 7. & $\begin{array}{l}\text { java-jar GenomeAnalysisTK.jar -R ucsc.hg 19.fasta-T HaplotypeCaller-I step7.bam -S STRICT-dbsnp } \\
\text { dbsnp_138.hg19.vcf-minPruning 3-o step8.vcf-stand_call_conf 50-stand_emit_conf } 30\end{array}$ & Calling variants in sequence data \\
\hline Step 8. & $\begin{array}{l}\text { java -jar GenomeAnalysisTK.jar -R ucsc.hg 19.fasta-TSelectVariants -variant step8.vcf-o step9_SNP.vcf } \\
\text {-selectType SNP -S STRICT }\end{array}$ & Select SNPs from the input file \\
\hline \multirow[t]{2}{*}{ Step 9.} & 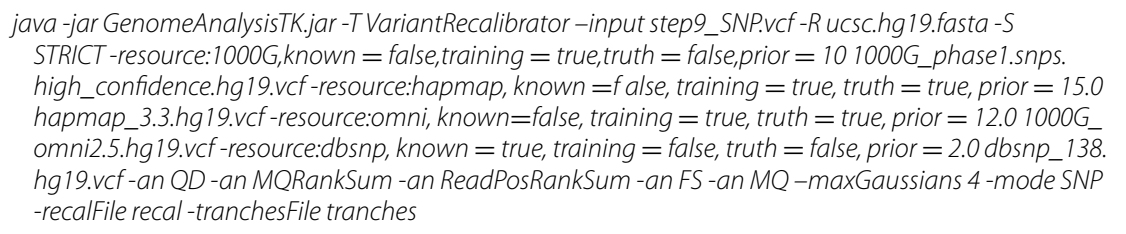 & Building SNP recalibration model \\
\hline & $\begin{array}{l}\text { java -jar GenomeAnalysisTK.jar-R ucsc.hg 19.fasta-T ApplyRecalibration-S STRICT-input step9_SNP.vcf } \\
\text {-ts_filter_level 99.5-mode SNP-tranchesFile tranches-recalFile recal-o step10_final.vcf }\end{array}$ & Applying SNP recalibration model \\
\hline
\end{tabular}

by increased number of polymorphic sites. This influenced an increased number of false calls accounted for by a presence of at least one alternative allele observed in BAM files of other mapping programs. LifeScope, albeit fully utilizing available multicore architecture, also had long running time. Another shortcoming of LifeScope is a lack of standalone tools similar to FastQC allowing to perform initial quality analysis of sequenced reads compressed in XSQ files.

\section{Conclusions}

We provided a comprehensive study of family exomes obtained by AB SOLiD 5500 platform and contributed technical details of the used pipelines. Our performed analysis strongly suggests that LifeScope's proprietary mapping program is the best choice for processing colorspace coded data generated by AB SOLiD platform. Although our conclusion was anticipated we nevertheless provided quantitative analysis to support it. In all cases LifeScope's pipeline stood out in terms of achieving high coverage and providing high confidence variants.

Coverage is one of the most important factors in calling a variant. Variant calls with low coverage might not indicate a true variant in the exome. Nevertheless a high coverage not necessarily indicates a true variant as well. A caveat was encountered in interpreting high coverage variants detected by SHRiMP-GATK approach. Larger than expected amount of high coverage singleton variants identified by SHRiMP-GATK that did not appear in the dbSNP database of known variants was observed. 
This prompted to a variant assessment strategy by exploring mappings (pileups [27]) in alternative BAM files at the genomic positions in which an unknown singleton SHRiMP-GATK variant was detected. We tested if a singleton variant is supported by the evidence of a presence of at least one alternative allele in that position in at least two other mappings. If it was not, the variant was filtered out.

This study was intended to explore near-default settings of the mapping programs, therefore a parameter optimization was not attempted. Aforementioned variant assessment strategy was applied only to SHRiMP-GATK variants since other pipelines did not deviate considerably from the expected small counts of singleton variants that are not present in dbSNP. In this study we used adjusted set of SHRiMP-GATK variants.

Our study reveals practical benefits of aggregating variant calling results of several pipelines. First of all variants that were identified by several methods have higher confidence of being truly present in the exome. If in doubt whether to consider the variant for further analysis because of its poorer mapping quality and coverage, one can gain confidence by examining mapping profile of the same genomic position in the alignment generated by some alternative mapping program. In summary:

- LifeScope's proprietary pipeline is method of choice for analysis of color-space coded sequencing data generated by AB SOLiD 5500 platform. LifeScope provided superior coverage of the exome sequencing data.

- Confirmation. Use of alternative pipelines may help in assessing an insufficiently covered variant and increasing the confidence about this identified variant truly being present in the exome.

- Sensitivity. Uniting lists of variants identified by several alternative mapping and variant calling pipelines allows to identify important deleterious variants that might have been missed by a single method because of poor coverage or mapping quality at that position.

- Choice of coverage threshold should be data-driven. Coverage of variants is not uniform. Due to this reason setting the high threshold on coverage may induce a considerable loss of important variants that may be strongly related to the manifestation of a phenotype of interest.

\section{Methods}

A workflow of exome analysis by LifeScope and the alternative pipeline is presented in Fig. 1. The raw exome data obtained from the sequencer in XSQ format is aligned to the reference genome by LifeScope mapping program. Alternatively, the XSQ files are converted to color-space fastq, csfasta and quality files and subsequently are aligned to the reference genome by MAQ, SHRiMP and BFAST mapping programs. In this way one exome is represented by four BAM files generated by four alignment tools. Variant calling is performed by two methods: LifeScope's diBayes algorithm and Genome Analysis Toolkit (GATK).

\section{LifeScope computational pipeline}

Exome analysis by Life Technologies ${ }^{\mathrm{TM}}$ LifeScope $^{\mathrm{TM}} 2.5 .1$ genomic analysis software was carried out in three stages described as primary, secondary and tertiary analyses [13]. Primary analysis of image acquisition and bead processing, application of quality metrics and color calls was performed within the SOLiD sequencer. Secondary and tertiary analyses were performed by LifeScope software using targeted.resequencing.frag workflow. This workflow consists of 7 LifeScope software modules. SAET, Mapping and Mapping statistics (BAMStats) are secondary analysis modules. Modules of tertiary analysis are Enrichment, SNPs, Small indels and Annotations. Default parameter values were used in LifeScope analysis as shown in Table 8.

\section{Alternative pipeline to the LifeScope}

Components of a pipeline alternative to the LifeScope are summarized in Table 9. The sequenced exomes in the SOLiD's XSQ file format were transformed to the color-space fastq format by XSQTools and BFAST solid2 fastq converters. Transformed reads were mapped to human hg19 reference genome by three alignment programs MAQ [29], SHRiMP [9] and BFAST [30]. Inputs for MAQ were color-space csfasta and quality qual files. SHRiMP and BFAST alignment programs used colorspace fastq files as inputs. SHRiMP and BFAST were chosen because of their ability to utilize a multiple-core architecture of available computational resources. The MAQ aligner was included because of its stringency and mapping accuracy reported in benchmarks [31]. By allowing three mismatches in MAQ a number of mapped reads increased by up to $10 \%$ at the expense of a threefold mapping time increase. In SHRiMP the default value of $-\mathrm{h}$ parameter controlling the quality of mapping window was changed to $85 \%$. In BFAST default parameter values were used. Variant calling was performed by Genome Analysis Toolkit (GATK) pipeline using BAM files generated by LifeScope, MAQ, SHRiMP and BFAST aligners as input. Variant calling workflow by GATK is presented in Table 7.

\section{Algorithmic details of the mapping programs}

Programs mapping next generation sequencing reads to the reference genome are subdivided into several 
Table 8 Parameters in LifeScope's secondary and tertiary analysis software modules

\begin{tabular}{|c|c|}
\hline & $\begin{array}{l}\text { Default } \\
\text { value }\end{array}$ \\
\hline \multicolumn{2}{|l|}{ Alignment parameters } \\
\hline Minimum number of non-matches allowed during indel finding & 9 \\
\hline Maximum deletion size (in a gapped alignment in the first pass) & 19 \\
\hline Maximum insertion size (in a gapped alignment in the first pass) & 4 \\
\hline The minimum edge length required for insertions and deletions on the first pass & 12 \\
\hline Number of mismatches allowed for gap alignments & 3 \\
\hline Minimum mapping quality value (MAPQ) allowed for aligned read & 8 \\
\hline Minimum edge length required for insertions and deletions & 12 \\
\hline The seed window side allowed to the left of the anchor alignment & 40 \\
\hline The seed window side allowed to the right of the anchor alignment & 80 \\
\hline $\begin{array}{l}\text { Maximum number of alignments for a read on the first pass which gives the maximum number of hits that } \\
\text { are reported in the mapping output }\end{array}$ & 50 \\
\hline \multicolumn{2}{|l|}{ SNPs analysis module using diBayes algorithm variant calling parameters } \\
\hline Minimum allele ratio (Het) & 0.15 \\
\hline Minimum coverage (Het) & 2 \\
\hline Minimum non-reference base QV (Het) & 28 \\
\hline Minimum average non-reference base QV (Hom) & 28 \\
\hline Minimum base quality value for a position & 28 \\
\hline Minimum base quality value of the non-reference allele of a position & 28 \\
\hline Mapping quality value of the read & $>8$ \\
\hline SNP call stringency. Alleles on both strands & Not required \\
\hline Threshold of mismatch/alignment-lengh ratio & 1 \\
\hline Base candidate allele quality value & $>7$ \\
\hline Minimum number of unique start positions required to call heterozygote, homozygote & 2 \\
\hline Proportion of the total reads containing either of the two candidate alleles & 0.65 \\
\hline
\end{tabular}

Table 9 List of open source tools used in our study of the alternatives to the LifeScope pipeline

\begin{tabular}{|c|c|c|}
\hline Analysis category & Tool & Web reference \\
\hline Convert XSQ to color-space fastq & XSQtools solid2fastq & Life Technologies website Bfast package \\
\hline \multirow[t]{6}{*}{ Alignment to the reference genome } & Maq v.0.7.1 & http://maq.sourceforge.net/ \\
\hline & Default parameters, -n 3 & \\
\hline & Shrimp 2.2 .3 & http://compbio.cs.toronto.edu/shrimp/ \\
\hline & Default parameters, -h 85 -strata -o 3 & \\
\hline & Bfast 0.7.0 & http://sourceforge.net/projects/bfast/ \\
\hline & Default parameters & \\
\hline \multirow[t]{2}{*}{ Preprocessing } & Picard 1.111 & http://picard.sourceforge.net/ \\
\hline & Samtools 0.1.18 & http://samtools.sourceforge.net/ \\
\hline \multirow[t]{2}{*}{ Variant calling } & GATK v.3.1 & http://www.broadinstitute.org/gatk/ \\
\hline & Steps and parameters in Table 7 & \\
\hline \multirow[t]{2}{*}{ Variant summaries } & Vcftools 0.1 .12 & http://vcftools.sourceforge.net/ \\
\hline & Bcftools v.0.2.0 & http://samtools.github.io/bcftools/ \\
\hline Variant annotation & Annovar & http://www.openbioinformatics.org/annovar/ \\
\hline
\end{tabular}

categories depending on how indexing and string matching are organized. They consist of algorithms that use a hash table (MAQ, BFAST, SHRiMP) and algorithms that use suffix trees (Burrows Wheeler Algorithm) [32]. Mapping programs used in the current study use hash tables for indexing of color-space encoded reference. MAQ 
encodes the reference genome into internal format. In the alignment phase MAQ indexes the reads. BFAST, SHRiMP and LifeScope index the reference genome using spaced seeds. All mappers perform gapped alignment, essential for a discovery of single nucleotide variations. In alignment of reads BFAST and SHRiMP at first finds a global list of candidate alignment locations for each read. During a second pass, the Smith-Waterman algorithm is applied locally on the identified candidate locations to find the best hits matching the reads [8]. Scholarly treatment of dynamic programming algorithm for sequence alignment that underlies local sequence alignment in used mapping programs can be found in the reference [33] on pages 172-176. In LifeScope mapping program a global indel finding extension option is used for alignment, which extends from anchor alignment and does a full length gapped extension with the allowed number of mismatches (set to 3) [13].

\section{Abbreviations}

BFAST: Blat-like Fast Accurate Search Tool; COSMIC: Catalog of Somatic Mutations in Cancer; DP: depth of coverage; GATK: Genome Analysis Tool Kit; MAQ: Mapping and Assembly with Qualities; NGS: Next Generation Sequencing; QUAL: Quality; SHRiMP: Short Read Mapping Package; SNP: Single Nucleotide Polymorphysms; Ti: transition; Tv: transversion.

\section{Authors' contributions}

VK and EP conceived the study. EP implemented alternative computational pipeline and summarized findings. TR implemented and tested computational steps by GATK and performed variant annotation by Annovar. AP performed exome analysis by LifeScope pipeline. EP and TR wrote the article. VK coordinated the project in which the exomes were sequenced. All authors read and approved the final manuscript.

\section{Acknowledgements}

This study is part of the LITGEN Project (VP1-3.1-MM-07-K-01-013) funded by the European Social Fund under the Global Grant Measure.

\section{Compliance with ethical guidelines}

\section{Competing interests}

The authors declare that they have no competing interests.

Received: 24 January 2015 Accepted: 24 August 2015

Published online: 07 September 2015

\section{References}

1. Coonrod EM, Durtschi JD, Margraf RL, Voelkerding KV. Developing genome and exome sequencing for candidate gene identification in inherited disorders: an integrated technical and bioinformatics approach. Arch Pathol Lab Med. 2013;137(3):415-33.

2. Kiezun A, Garimella K, Do R, Stitziel NO, Neale BM, McLaren PJ, Gupta N, Sklar P, Sullivan PF, Moran JL, Hultman CM, Lichtenstein P, Magnusson P, Lehner T, Shugart YY, Price AL, de Bakker PI, Purcell SM, Sunyaev SR. Exome sequencing and the genetic basis of complex traits. Nat Genet. 2012;44(6):623-30.

3. D'Antonio M, D'Onorio De Meo P, Paoletti D, Elmi B, Pallocca M, Sanna N, Picardi E, Pesole G, Castrignano T. WEP: a high-performance analysis pipeline for whole-exome data. BMC Bioinform. 2013;14(Suppl 7):S11.
4. Fischer M, Snajder R, Pabinger S, Dander A, Schossig A, Zschocke J, Trajanoski Z, Stocker G. SIMPLEX: cloud-enabled pipeline for the comprehensive analysis of exome sequencing data. PLoS One. 2012;7(8):e41948.

5. Brownstein CA, Beggs AH, Homer N, Merriman B, Yu TW, Flannery KC, DeChene ET, Towne MC, Savage SK, Price EN, Holm IA, Luquette LJ, Lyon E, Majzoub J, Neupert P, McCallie D Jr, Szolovits P, Willard HF, Mendelsohn NJ, Temme R, Finkel RS, Yum SW, Medne L, Sunyaev SR, Adzhubey I, Cassa CA, de Bakker PI, Duzkale H, Dworzyński P, Fairbrother W, et al. An international effort towards developing standards for best practices in analysis, interpretation and reporting of clinical genome sequencing results in the CLARITY Challenge. Genome Biol. 2014;15(3):R53.

6. Lampa S, Dahlo M, Olason PI, Hagberg J, Spjuth O. Lessons learned from implementing a national infrastructure in Sweden for storage and analysis of next-generation sequencing data. Gigascience. 2013;2(1):9.

7. Mazza T, Castellana S. Multi-Sided compression performance assessment of ABI SOLiD WES data. Algorithms. 2013;6:309-18.

8. Homer N, Merriman B, Nelson SF. Local alignment of two-base encoded DNA sequence. BMC Bioinform. 2009;10:175

9. David M, Dzamba M, Lister D, Ilie L, Brudno M. SHRiMP2: sensitive yet practical short read mapping. Bioinformatics. 2011;27(7):1011-2.

10. Pabinger S, Dander A, Fischer M, Snajder R, Sperk M, Efremova M, Krabichler B, Speicher MR, Zschocke J, Trajanoski Z. A survey of tools for variant analysis of next-generation genome sequencing data. Brief Bioinform. 2014;15(2):256-78.

11. Fonseca NA, Rung J, Brazma A, Marioni JC. Tools for mapping highthroughput sequencing data. Bioinformatics. 2012;28(24):3169-77.

12. Castellana S, Romani M, Valente EM, Mazza T. A solid quality-control analysis of AB SOLiD short-read sequencing data. Brief Bioinform. 2013;14(6):684-95.

13. LifeScope ${ }^{\text {TM }}$. Users manual, Lifescope Genomic Analysis Software 2.5.1, Command Shell, DATA ANALYSIS METHODS AND INTERPRETATION. Publication Part Number 4476538 Rev. A. 2012.

14. Rancelis T, Cimbalistiene L, Kucinskas V. Next-generation whole-exome sequencing contribution to identification of rare autosomal recessive diseases. Acta Medica Lith. 2013;20(1):43-51.

15. Wang K, Li M, Hakonarson H. ANNOVAR: functional annotation of genetic variants from high-throughput sequencing data. Nucleic Acids Res. 2010;38(16):e164.

16. Rancelis T, Pranckeviciene E, Kucinskas V. Annotation tools and computer programs for genome/exome data analysis. Lab Med t.15 (in Lithuanian). 2013;4(60):206-12.

17. DePristo MA, Banks E, Poplin R, Garimella KV, Maguire JR, Hartl C, Philippakis AA, del Angel G, Rivas MA, Hanna M, McKenna A, Fennell TJ, Kernytsky AM, Sivachenko AY, Cibulskis K, Gabriel SB, Altshuler D, Daly MJ. A framework for variation discovery and genotyping using next-generation DNA sequencing data. Nat Genet. 2011;43(5):491-8.

18. McKenna A, Hanna M, Banks E, Sivachenko A, Cibulskis K, Kernytsky A, Garimella K, Altshuler D, Gabriel S, Daly M, DePristo MA. The Genome Analysis Toolkit: a MapReduce framework for analyzing next-generation DNA sequencing data. Genome Res. 2010;20(9):1297-303.

19. de Ligt J, Boone PM, Pfundt R, Vissers LELM, de Leeuw N, Shaw C, Brunner HG, Lupski JR, Veltman JA, Hehir-Kwa JY. Platform comparison of detecting copy number variants with microarrays and whole-exome sequencing. Genom Data. 2014;2:144-6.

20. Gregor A, Oti M, Kouwenhoven EN, Hoyer J, Sticht H, Ekici AB, Kjaergaard S, Rauch A, Stunnenberg HG, Uebe S, Vasileiou G, Reis A, Zhou H, Zweier C. De novo mutations in the genome organizer CTCF cause intellectual disability. Am J Hum Genet. 2013;93(1):124-31.

21. Ebersberger I, Metzler D, Schwarz C, Paabo S. Genomewide comparison of DNA sequences between humans and chimpanzees. Am J Hum Genet. 2002;70(6):1490-7.

22. Guo Y, Long J, He J, Li Cl, Cai Q, Shu XO, Zheng W, Li C. Exome sequencing generates high quality data in non-target regions. BMC Genom. 2012;13:194.

23. Quinlan AR. BEDTools: The Swiss-Army Tool for Genome Feature Analysis. Curr Protoc Bioinform. 2014;47:1-11.

24. O'Rawe J, Jiang T, Sun G, Wu Y, Wang W, Hu J, Bodily P, Tian L, Hakonarson H, Johnson WE, Wei Z, Wang K, Lyon GJ. Low concordance of multiple variant-calling pipelines: practical implications for exome and genome sequencing. Genome Med. 2013;5(3):28. 
25. Landrum MJ, Lee JM, Riley GR, Jang W, Rubinstein WS, Church DM, Maglott DR. ClinVar: public archive of relationships among sequence variation and human phenotype. Nucleic Acids Res. 2014;42:D980-5.

26. Forbes SA, Beare D, Gunasekaran $P$, Leung $K$, Bindal N, Boutselakis H, Ding M, Bamford S, Cole C, Ward S, Kok CY, Jia M, De T, Teague JW, Stratton MR, McDermott U, Campbell PJ. COSMIC: exploring the world's knowledge of somatic mutations in human cancer. Nucleic Acids Res. 2015;43:D805-11.

27. Li H, Handsaker B, Wysoker A, Fennell T, Ruan J, Homer N, Marth G, Abecasis G, Durbin R. The Sequence Alignment/Map format and SAM tools. Bioinformatics. 2009;25(16):2078-9.

28. Quinlan R. C4.5: Programs for Machine Learning. San Francisco: Morgan Kaufmann Publishers Inc.; 1993.
29. Li H, Ruan J, Durbin R. Mapping short DNA sequencing reads and calling variants using mapping quality scores. Genome Res. 2008;18(11):1851-8.

30. Homer N, Merriman B, Nelson SF. BFAST: an alignment tool for large scale genome resequencing. PLoS One. 2009;4(11):e7767.

31. Hatem A, Bozda D, Toland AE, Catalyurek UV. Benchmarking short sequence mapping tools. BMC Bioinform. 2013;14:184.

32. Li H, Homer N. A survey of sequence alignment algorithms for nextgeneration sequencing. Brief Bioinform. 2010;11(2):473-83.

33. Lesk AM. Introduction to bioinformatics. New York: Oxford University Press; 2002

\section{Submit your next manuscript to BioMed Central} and take full advantage of:

- Convenient online submission

- Thorough peer review

- No space constraints or color figure charges

- Immediate publication on acceptance

- Inclusion in PubMed, CAS, Scopus and Google Scholar

- Research which is freely available for redistribution

Submit your manuscript at

www.biomedcentral.com/submit

C Biomed Central 